Z. Klin. Chem. Klin. Biochem.

11. Jg. 1973 , S. $259-262$

\title{
Zur Methodik der Bestimmung der ETR (Effective Thyroxine Ratio)
}

\author{
Von K. H. RudorfF*), J. HerRmanN und H. L. KRÜSKEMPER \\ 2. Mediqinische Universitätsklinite Düsseldorf; \\ Abt. für Klin. Endokrinologie*) am Dept. Innere Medizin der Medizinischen Hochschule Hannover
}

(Eingegangen am 14. März/17. Mai 1973)

Herrn Professor Dr. Dr. E. Schütte zum 65. Geburtstag genvidmet

\begin{abstract}
Es wird über die Effective Thyroxine Ratio (ETR), einen Test zur Beurteilung der Schilddrüscnfunktion, berichtet, bei dem die Bestimmung der Thyroxinkonzentration im Testserum unter Anwesenheit von Bindungsprotcinen desselben Testserums durchgeführt wird. Die ETR wird berechnet aus der Thyroxinkonzentration des Testserums und eines normalen Poolserums.

Die Untersuchungen an euthyreoten Versuchspersonen (Normalpersonen, Schwangere, Frauen unter hormonellen Kontrazeptiva, Patienten mit akuter Hepatitis), bei Hyperthyreose und Hypothyreose zeigen, daß die ETR ein geeigneter Parameter für die klinische Routine zur Beurteilung der Schilddrüsenfunktion ist, da sie Auskunft gibt über die freie, stoffwechselaktive $T_{4}-F r a k t i o n$. Die Vorteile der hier beschriebenen Modifikation des kommerziell erhältlichen Tests liegt in der Einfachheit, in dem geringen Zeitaufwand und vor allem in den niedrigen Kosten.
\end{abstract}

\section{Determination of the Effective Thyroxin Ratio (ETR)}

The Effective Thyroxine Ratio (ETR) was investigated as an assessment of thyroid function. The concentration of thyroxine in the test serum is measured in the presence of thyroxine-binding proteins of the same test serum. ETR is calculated as the ratio of the concentrations of thyroxine in the test serum and in normal pool serum. From measurements in patients with normal thyroid function (i. e. normal subjects, pregnant women, women on oral contraceptives and patients with acute hepatitis) and in patients with either hyper- oder hypothyroidism ETR has proven to be a valid parameter for the assessment of thyroid function. Advantages of this method are its independence from the concentration of thyroxine binding protein, its simplicity and rapidity, and its low costs.

Die beiden zur Zeit gebräuchlichsten Serumparameter zur quantitativen Beurteilung der Schilddrüsenfunktion - das Gesamtthyroxin (oder proteingebundenes ${ }^{127} \mathrm{Jod}$ ) und der sogenannte Serum- ${ }^{131} \mathrm{~J}-\mathrm{T}_{3}-$ Test $^{1}$ ) können oft verfälscht werden durch abnorme Konzentrationen an thyroxinbindenden Proteinen (akute Hepatitis, Schwangerschaft, genetisch bedingter Mange] oder Überschuß an thyroxinbindendem Globulin, Nephrose, Akromegalie, dekompensierte Lebercirthose, Östrogene, Androgene, Corticoide, Salicylate, Phenylbutazon, Diphenylhydantoin, Sulfonylharnstoffe u. a.). Bei Vorliegen solcher" abnormer Bindungsverhältnisse für Thyroxin stellt die Bestimmung der Konzentration des stoffwechselaktiven freien Thyroxins oft den entscheidenden Parameter dar. Die übliche Methodik ist jedoch für den Routinebetrieb sehr aufwendig und zeitraubend. Der freie $T_{4}$-Index (1), das Produkt aus ,, $\mathrm{T}_{3}$ in vitro uptake " und Serum-Gesamt- $\mathrm{T}_{4}$ (oder $\mathrm{PB}^{127} \mathrm{~J}$ ), der auch bei Vorliegen von Bindungsabnormitāten gut mit der Schilddrüsenfunktion korreliert, ist ebenfalls für den Routinebetrieb nicht geeignet, da zwei verschiedene Bestimmungsmethoden mit zahlreichen Arbeitsgängen durchgeführt werden müssen. Ziel dieser Arbeit ist es, eine Modifikation des bereits kommerziell erhältlichen nẹen Tests - die

1) Abkürzungen: $\mathrm{T}_{3}=$ Trijodthyronin; $\mathrm{T}_{4}=$ Thyroxin; $\mathrm{PB}^{122} \mathrm{~J}=$ proteingebundenes ${ }^{127} \mathrm{Jod}$; TBG $=$ Thyroxin bindendes Globulin.
„Effective Thyroxine Ratio“ $(2,3)-$, mit der indirekt Auskunft über die stoffwechselaktive freie Hormonfraktion erhalten werden kann, auf ihre klinische Anwendbarkeit, ihre Unabhängigkeit von Bindungsproteinen und hinsichtlich Ubertragbarkeit auf unser Testsystem für die Bestimmung von Gesamtthyroxin (4) zu untersuchen.

\section{Material und Methoden}

Das Thyroxin im Serum wurde nach der Vorschrift von Murphy und Patree in der Modifikation nach Siersbaek-Nielsen (5) unter Verwendung von Dowex 1 X 8 200-400 mesh als Ionenaustauscher bestimmt (4). Das radioaktive ${ }^{125} \mathrm{~J}$-Thyroxin wurde vom Radiochemical Center, Amersham, bezogen. Für jeden Bestimmungstag wurde eine volle Standardkurve mit $5 \mathrm{Meß}$ punkten als Doppelwerte festgelegt. Die Äthanolextraktion des Thyroxins aus dem Serum erfolgte durch Hinzufügen von $2 \mathrm{ml}$ 95proz. Äthanol zu $1 \mathrm{ml}$ Serum und anschließendes Mischen für $30 \mathrm{~s}$ und Zentrifugieren für etwa $3 \mathrm{~min}$ bei $2000 \mathrm{U} / \mathrm{min} .0,3 \mathrm{ml}$ Uberstand wurden zur Trockene eingedampft. Zur Bestimmung der „Effective Thyroxine Ratio" (ETR) wurden $5 \mu \mathrm{l}$ des Testserums an die Wand des Teströhrchens getropft und danach zusammen mit dem eingedampften Extrakt in $1 \mathrm{ml}$ radioaktiver $\mathrm{T}_{4}$-TBG-Lösung aufgenommen und gut geschüttelt. Die weitere Aufarbeitung der Proben entsprach dem Vorgehen bei der Bestimmung des Thyroxins (4,5) (Abb. 1). Die ETR wurde aus folgender Formel berechnet

$$
\mathrm{ETR}=\frac{\mathrm{Imp} \cdot / \mathrm{min}_{\text {Pool-Serum }}}{\mathrm{Imp} \cdot / \mathrm{min}_{\text {Patlenten-Serum }}} .
$$

Die Bestimmung des freien Thyroxins im Serum erfolgte nach HerrmanN und KrüsKEMPER (6). 


\section{Ergebnisse}

In Tabelle 1 und Abbildung 1 sind die Ergebnisse der ETR, verglichen mit der Serum- $\mathrm{T}_{4}$-Konzentration, für verschiedene Patientengruppen zusammengestellt. Die

Tab. 1

Serum- $T_{4}$-Konzentration und Effective Thyroxine Ratio bei Euthyreose, Hyper- und Hypothyreose und Euthyreose mit z. T. veränderten $T_{4}$-Bindungsproteinen. $\bar{x} \pm 2 \mathrm{~s}$; in Klammern Anzahl der untersuchten Patienten. +) $\mathrm{p}<0,0005$ gegenüber Euthyreose

\begin{tabular}{lcc}
\hline & $\begin{array}{c}\text { Serum-T, }- \text { Konzentration } \\
{[\mu \mathrm{g} / \mathrm{l}]}\end{array}$ & $\begin{array}{c}\text { Effective Thyroxine Ratio } \\
\text { (ETR) }\end{array}$ \\
\hline Euthyreose & $84,3 \pm 15,8(\mathrm{n}=97)$ & $0,914 \pm 0,097(\mathrm{n}=97)$ \\
Hyperthyreose & $183,1 \pm 43,1(\mathrm{n}=39)$ & $\left.1,613^{+}\right) \pm 0,389(\mathrm{n}=39)$ \\
Hypothyreose & $28,8 \pm 22,6(\mathrm{n}=44)$ & $\left.0,651^{+}\right) \pm 0,094(\mathrm{n}=44)$ \\
Euthyreose mit $\mathrm{z}$. T. veränderten & \\
T,-Bindungsproteinen: & \\
Gravidität & $121,0 \pm 22,8(\mathrm{n}=15)$ & $0,901 \pm 0,072(\mathrm{n}=15)$ \\
Hormonelle & & \\
Kontrazeptiva & $109,4 \pm 13,7(\mathrm{n}=13)$ & $0,966 \pm 0,095(\mathrm{n}=13)$ \\
Akute Hepatitis & $119,8 \pm 25,9(\mathrm{n}=12)$ & $0,933 \pm 0,098(\mathrm{n}=12)$ \\
Nephrose & 68,0 & 0,990 \\
Plasmocytom & 102,0 & 0,900 \\
Plasmocytom & 163,0 & 0,880 \\
Plasmocytom & 74,0 & 0,830 \\
Morbus BECHTEREw & 140,0 & 0,980 \\
Morbus BECHTEREW $: 156,0$ & 1,160 \\
NNR-Tumor & 85,0 & $0,92 n$ \\
NNR-Tumor & 81,0 & 0,860 \\
Colitis ulcerosa & 41,0 & 0,930 \\
Colitis ulcerosa & 89,0 & 0,830 \\
\hline
\end{tabular}

97 euthyreoten Probanden zeigen ETR-Werte zwischen 0,817 und 1,011 (1 s-Bereich). Im Gegensatz $\mathrm{zu}$ den entsprechenden Gesamt- $\mathrm{T}_{\mathbf{4}}$-Daten liegen, alle ETR-Werte von euthyreoten Patienten (Hepatitis, Schwangerschaft, Frauen unter hormonellen Kontrazeptiva, Nephrosen, Plasmocytom, Morbus BechteREw, NNR-Tumor, Colitis ulcerosa) in dem für euthyreote Personen bestimmten Normalbereich. Dagegen liegen die Werte bei Hyper- und Hypothyreose hochsignifikant $(p<0,0005)$ über bzw. unter denen der Euthyreose.

Die Standardabweichung der Doppelwerte der ETR betrug 0,041 ( $n=25)$. Der Variationskoeffizient bei der Bestimmung von „Tag zu Tag“ lag bei $\pm 2,3 \%$.

Abbildung 2 zeigt dịe Beziehung zwischen ETR und freiem Thyroxin im Serum bei 90 Patienten. Der Korrelationskoeffizient beträgt $r=0,9125$, ist also hochsignifikant: $2 \alpha<0,001 \quad(a=0,6539, b=0,1099$, $\left.s_{\mathbf{x} \cdot \mathbf{y}}= \pm 0,1004\right)$.

Abbildung 3 zeigt die Abhängigkeit der ETR-Werte von der zugesetzten Menge Testserum (5, 10, 50 und $100 \mu 1)$. Die Werte von euthyreoten Personen, von Patienten mit Hepatitis und Frauen unter hormoneller Kontrazeption ändern sich nur geringfügig. Der ETRWert bei Schwangeren fällt bei Zugabe von $10 \mu \mathrm{l}$ Serum deutlich $a b$, so daß er sich gegenüber der Hypothyreose nicht mehr signifikant unterscheidet. Gleich-

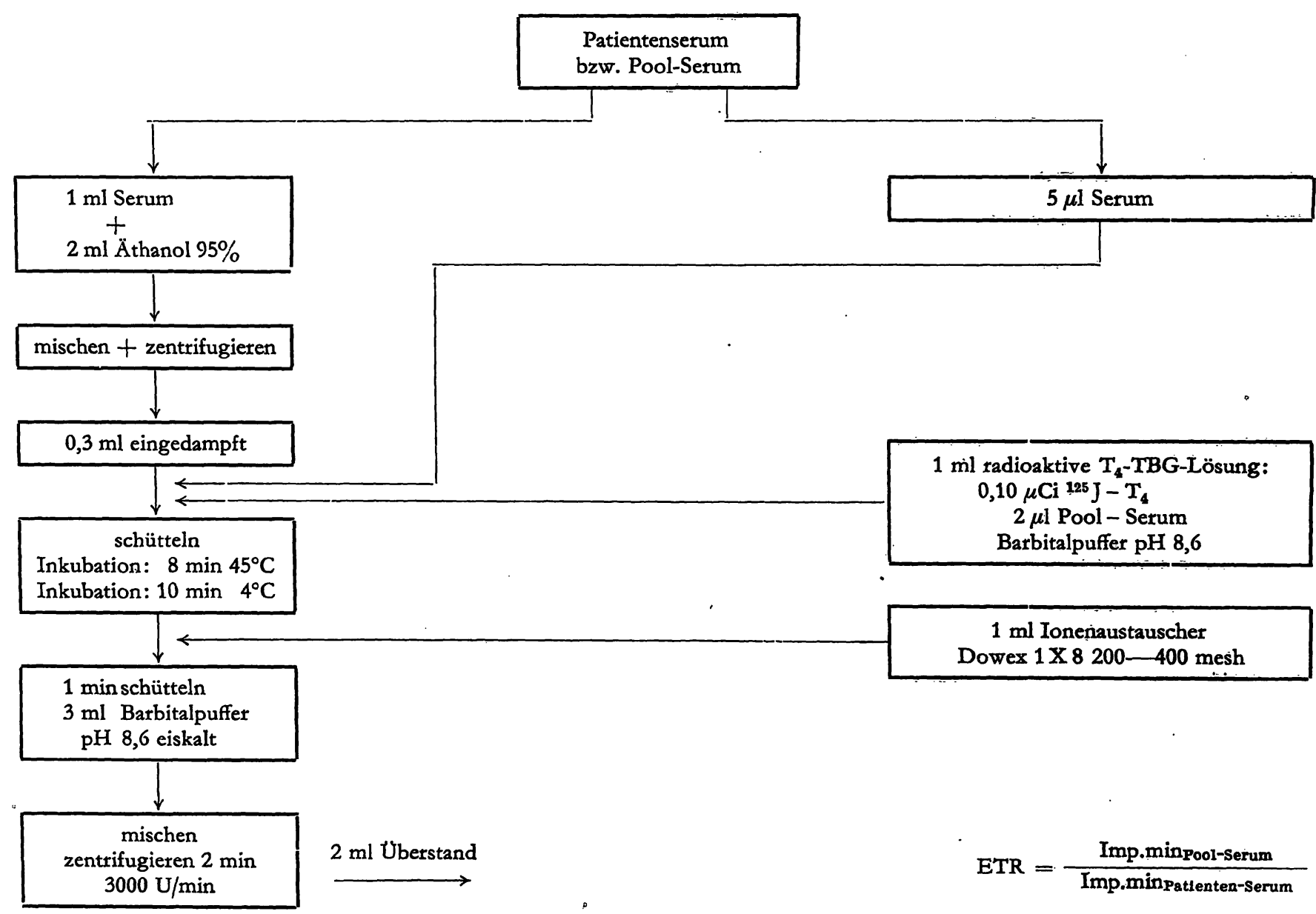

Abb. 1

Arbeitsgang zur Bestimmung der „Effective Thyroxine Ratio“. Ohne Zugabe von $5 \mu$ l Serum entspricht das Vorgehen der Bestimmung von Gesamt-Thyroxin im Serum 
Abb. 2

Vergleich zwischen Gesamt-T -Konzentration im $n$ - Anzahil der Versuche

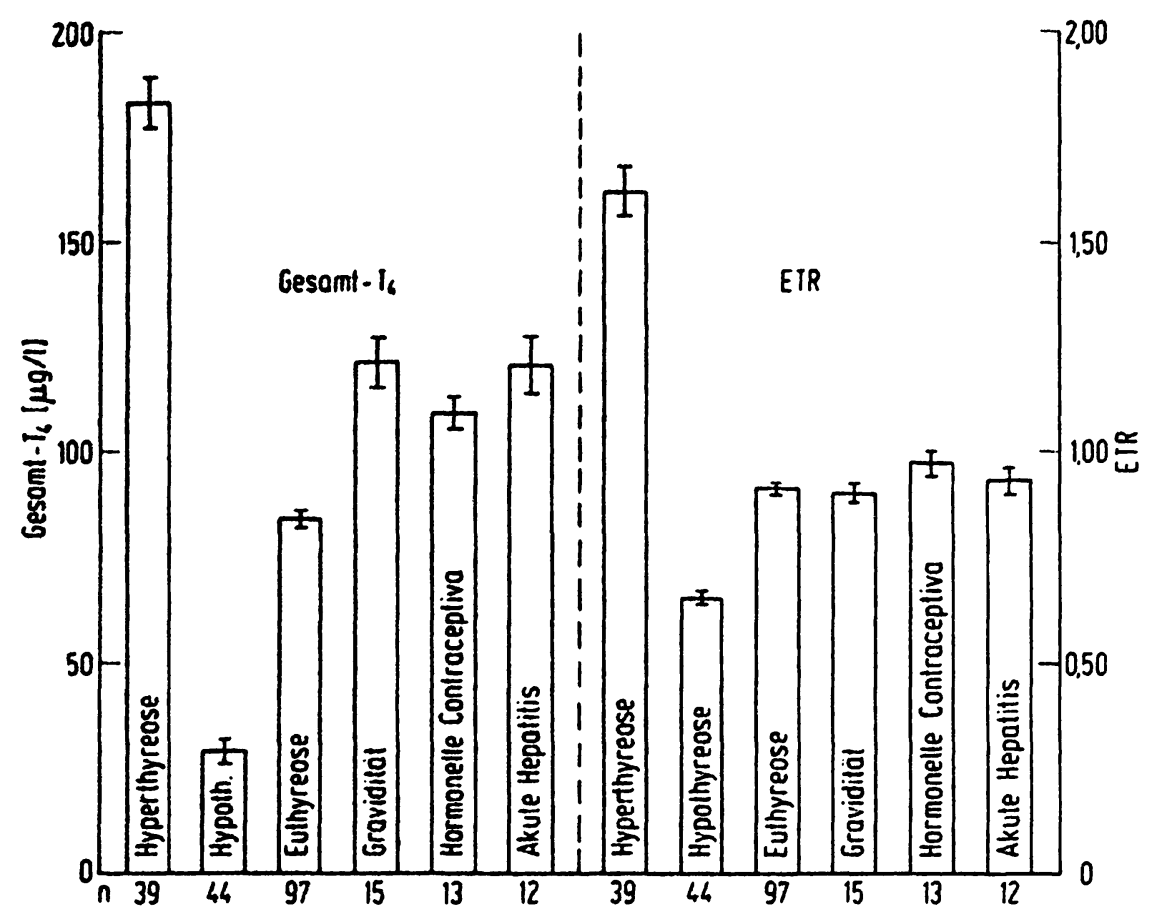

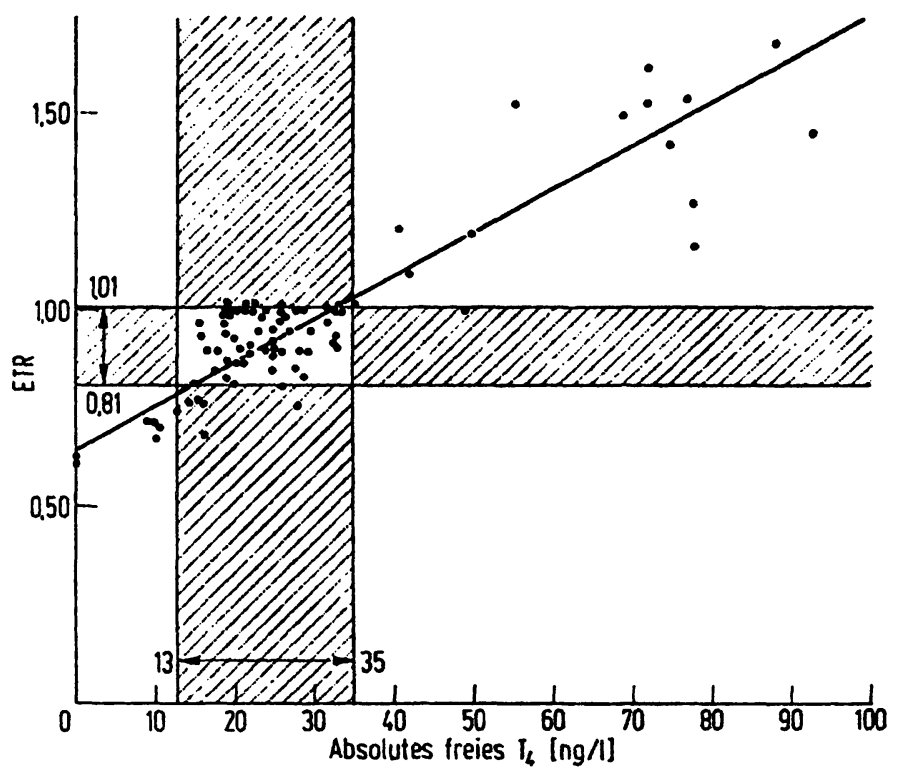

Abb. 3

Beziehung zwischen ETR und absolutem freien $T_{4}$ $r=0,9125 \quad a=0,6539 \quad b=0,1099$

zeitig steigt der ETR-Wert bei Hypothyreose an und ist bei Zugabe von $>10 \mu l$ Serum von den ETRWerten bei Euthyreose nicht mehr signifikant unterschiedlich.

\section{Diskussion}

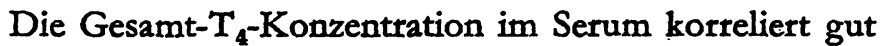
mit dem Schilddrüsenstatus, wenn keine Abnormalitäten der Bindungsproteine vorliegen. Bei Hepatitis, Schwangeren und Frauen unter hormoneller Kontrazeption u. a. kommt es zu Uberlappungen der Thyroxinwerte mit dem hyperthyreoten Bereich. Das Gesamtthyroxin ist in diesen Fällen als alleiniges Kriterium zur Beurteilung des Schilddrüsenstatus unzuverlāssig. Auch die sogenannten $T_{3}$-in-vitro-Tests

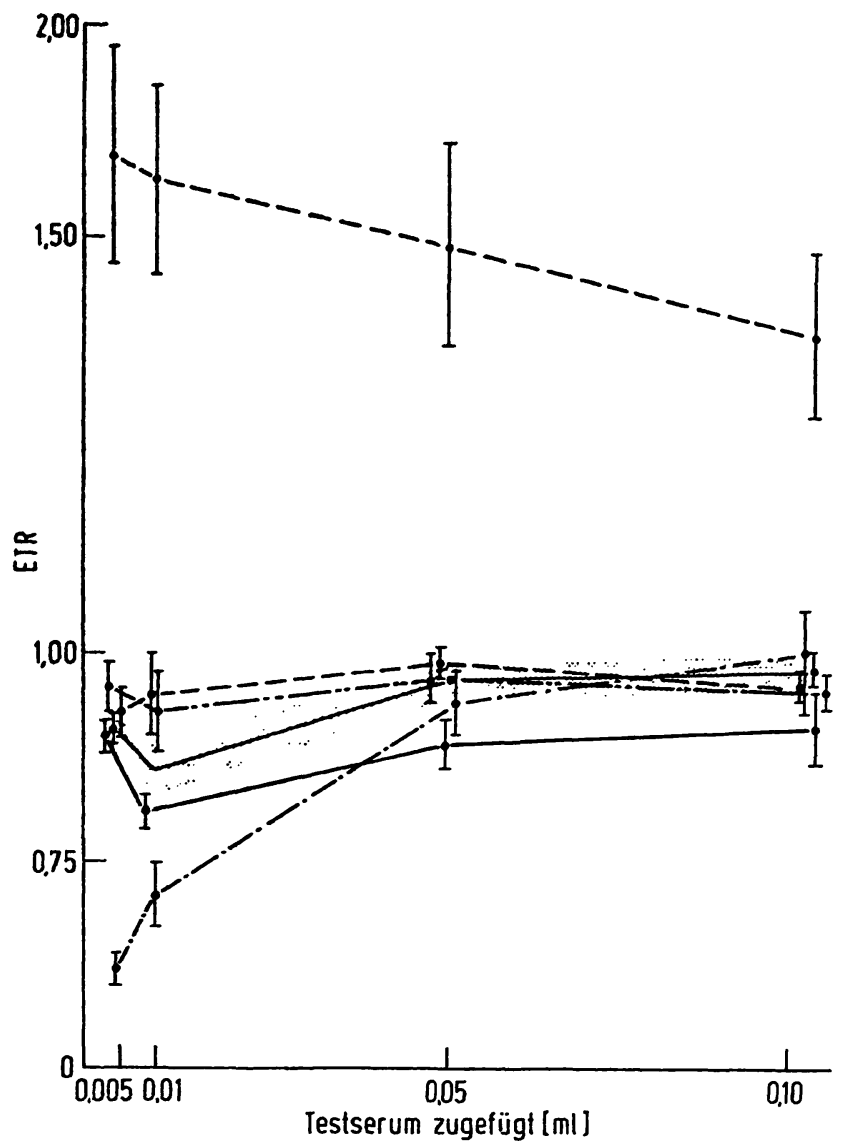

Abb. 4

Abhängigkeit der ETR-Werte vom zugefügten Volumen des Testserums. $\overline{\mathbf{x}} \pm s_{\bar{x}} \quad n=$ Anzahl der Versuche

Euthyreose $(n=13)$

Hyperthyreose $(n=13)$

- - Hypothyreose $(\mathrm{n}=13)$

- Schwangerschaft $(n=13)$

...- Hormonelle Kontrazeptiva $(n=13)$

sind als Suchtests unzureichend, da etwa $45 \%$ der hypothyreoten und 23\% der hyperthyreoten Patienten eine normale $\mathrm{T}_{3}$-Aufnahme und $89 \%$ der Schwangeren 
und mit Östrogenen behandelten Frauen eine erniedrigte $\mathrm{T}_{3}$-Aufnahme zeigen $(2,7)$.

Der Vorteil der in der vorliegenden Arbeit beschriebenen Bestimmung der ETR liegt darin, daß Veränderungen des Thyroxinspiegels, die auf pathologischen Funktionszuständen der Schilddrüse beruhen, deutlich zum Ausdruck kommen, während Änderungen des Thyroxinspiegels, die allein durch Änderungen der Bindungskapazität der Thyroxin bindenden Serumproteine bedingt sind, zu normalen Meßergebnissen führen, und somit eine normale Schilddrüsenfunktion anzeigen. Ein erhöhter Thyroxinspiegel bei der Hyperthyreose führt zunächst zu einer Verdrängung einer erhöhten Menge radioaktiven Thyroxins aus seiner ursprünglichen Bindung (competitive Proteinbindungsanalyse). Das freigesetzte radioaktive Thyroxin wird zu einem geringen Anteil an eine Probe desselben Patientenserums gebunden, da diese bereits durch den erhöhten endogenen Thyroxinspiegel weitgehend abgesättigt ist. Dem Ionenaustauscher steht somit eine erhöhte Menge an radioaktivem Thyroxin zur Verfügung.

Bei einem verminderten endogenen Thyroxinspiegel wird entsprechend weniger radioaktives Thyroxin freigesetzt, das vermehrt an der gering abgesättigten Probe desselben Serums angelagert wird. Es stehen somit nur geringe Mengen Thyroxin dem Ionenaustauscher zur Verfügung. Bei einer Vermehrung der Bindungsfähigkeit des Serums für Thyroxin z. B. in der Gravidität, unter hormonellen Kontrazeptiva usw., wird zwar im ersten Teil des Tests vermehrt radioaktives Thyroxin freigesetzt, aber dieses vermehrt freigesetzte Thyroxin wird nach Zugabe desselben Serums durch dessen vermehrte freie Bindungskapazität für Thyroxin auch vermehrt aufgenommen. Dem Ionenaustauscher steht demnach nur eine der Norm entsprechende Menge an radioaktivem Thyroxin zur Verfügung.

Aus den in Abbildung 3 dargestellten Untersuchungen geht hervor, daß für unser. Testsystem eine Zugabe von $5 \mu \mathrm{l}$ Serum am günstigsten ist, weil damit die ETRWërte aller Euthyreosen am dichtesten zusammenliegen und sich am deutlichsten von der Hyper- und Hypothyreose unterscheiden.

Abbildung 2 zeigt, daß eine gute Korrelation zwischen ETR und freiem Thyroxin im Serum besteht. THorson et al. (2) konnten zeigen, daß die Aussagekraft der ETR vergleichbar ist mit der des freien $T_{4}$-Index $\left(T_{3}\right.$-in vitro uptake-Serum-Gesamt- $\left.T_{4}\right)$. Der Vorteil der ETR-Bestimmung liegt darin, daß mit einem Test eine Aussage gemacht werden kann über die stoffwechselaktive Hormonfraktion. Weitere Vorteile sind die Einfachheit, der geringe Zeitaufwand und die niedrigen Kosten. So können in unserem Laboratorium an einem Tag 100 ETR-Bestimmungen durchgeführt werden. Damit ist die hier beschriebene Modifikation als Routinemethode im Laboratorium mit großem Probenanfall besonders geeignet. Gegenwärtig werden weitere Untersuchungen über die Aussagekraft der ETR über die Schilddrüsenfunktion bei Patienten, die unter Therapie mit Diphenylhydantoin, Sulfonylharnstoffen, Phenylbutazon, Salicylaten u. a. stehen und über die Eignung zur Verlaufskontrolle bei der Therapie von Schilddrüsenerkrankungen durchgeführt.

\section{Literatur}

1. Clark, F. \& Horn, D. B. (1965), J. Clin. Endocrinol. Metab. 25, 39-45. - 2. Thorson, S. C., Mincey, E. K., McIntosh, H. W. \& Morrison, R. T. (1972), Brit. Med. J. 2, 67-71. 3. Mincey, E. K., Thorson, S. C., Brown, J. L., Morrison, R. T. \& McIntosh, H. W. (1972), J. Nuclear Med. 13, 165-168.

4. HerramanN, J., Brase, A., de Marees, H. \& Krüskemper,
H. L. (1970), diese Z. 8, 605-610. - 5. Siersbaek-Nielsen, K. (1967), Acta Med. Scand. 181, 327. - 6. HerrmanN, J. \& KrüsKEMPER, H. L. (1971), diese Z. 9, 320-323. - 7. MrtChELL, M. L., Harden, A. B. \& O'Rourhe, M. E. (1960), J. Clin. Endocrinol. Metab. 20, 1474.
Priv. Doz. Dr. J. Herrmann 4000 Düsseldorf 1 Moorenstr. 5 